\title{
INVERSE IDENTIFICATION OF LOCAL STIFFNESS ACROSS ASCENDING THORACIC AORTIC ANEURYSMS
}

\author{
Solmaz Farzaneh • Olfa Trabelsi . \\ Stéphane Avril
}

the date of receipt and acceptance should be inserted later

\begin{abstract}
Aortic dissection is the most common catastrophe of the thoracic aorta, with a very high rate of mortality. Type A dissection is often associated with an ascending thoracic aortic aneurysm (ATAA). However, it is widely acknowledged that the risk of type A dissection cannot be reliably predicted simply by measuring the ATAA diameter and there is a pressing need for more reliable risk predictors. It was previously shown that there is a significant correlation between a rupture criterion based on the ultimate stretch of the ATAA and the local membrane stiffness of the aorta. Therefore, reconstructing regional variations of the membrane stiffness across the aorta appears highly important. In this paper, we present a novel non-invasive inverse method to identify the patient-specific local membrane stiffness of aortic walls based on preoperative gated CT scans. Using these scans, a structural mesh is defined across the aorta with a set of nodes attached to the same material points at different time steps throughout the cardiac cycle. For each node, time variations of the position are analyzed using Fourier series, permitting the reconstruction of the local strain distribution (fundamental term). Relating these strains to tensions with the membrane stiffness, and writing the local equi-

S. Farzaneh · O. Trabelsi S. Avril*

Mines Saint-Étienne, CIS-EMSE, F-42023 Saint-Étienne, France

INSERM, U1059, SAINBIOSE, F-42023 Saint-Étienne, France

Université de Lyon, F-69000 Lyon, France

${ }^{*}$ Corresponding author E-mail: avril@emse.fr
\end{abstract}


librium satisfied by the tensions, the local membrane stiffness is finally derived at every position. The methodology is applied onto the ascending and descending aorta of three patients. Interestingly, the regional distribution of identified stiffness properties appears heterogeneous across the ATAA. Averagely, the identified stiffness is also compared with values obtained using other non-local methodologies. The results support the possible non-invasive prediction of stretch-based rupture criteria in clinical practice using local stiffness reconstruction.

Keywords Non-invasive inverse method $\cdot$ Local membrane stiffness $\cdot$ Finiteelements · Ascending thoracic aortic aneurysms - Patient-specific - Risk of rupture

\section{Introduction}

Ascending thoracic aortic aneurysms (ATAAs) manifest by localized ballooning of the aorta. They are difficult to detect because they usually have no symptom. Unrecognized and untreated TAA may lead to dissection or rupture of the aneurysm 5 ending with instantaneous death. Independently of age and gender, about 30,000 6 people in Europe and 15,000 people in the United States are diagnosed with a TAA every year $[5,31,42]$.

$8 \quad$ ATAA are commonly treated with a timely surgical repair by replacement of 9 the dilated aortic segment with synthetic grafts. For patients without any famil- ial disorders such as Marfan syndrome, elective surgical intervention of ATAA is 11 recommended when its diameter is larger than $5.5 \mathrm{~cm}$ or when it is considered as a fast growing aneurysms (growth $>1 \mathrm{~cm}$ per year) $[6,8,9,15,31]$. The diameter 3 of $5.5 \mathrm{~cm}$ as a criterion for decision-making of surgical intervention is extensively 14 recognized as an insufficient criterion. For example, the International Registry of 5 Acute Aortic Dissection (IRAD) conveyed that among 591 type "A" aortic dis6 sections, $59 \%$ had a diameter below $5.5 \mathrm{~cm}$. Moreover, several studies considering abdominal aortic aneurysm (AAA) suggest that biomechanical factors may reliably predict the risk of rupture rather than the diameter criterion alone $[17,20$, 9 14]. Wall stress analysis and identification of patient-specific material properties of abdominal aortic aneurysms using 4D ultrasound were performed to improve rup${ }_{21}$ ture risk assessment [49]. It is well-known that the rupture of ATAA occurs when 
the stress applied to the aortic wall reaches the rupture stress [34,36,47]. Another definition of rupture can be represented when the stretch applied to the tissue is greater than its maximum extensibility or distensibility (stretch based rupture criterion). Accordingly, Duprey et al. [13] proposed a stretch-based rupture risk criterion and showed its correlation with tangent elastic modulus in ATAA based on data collected in 31 patients. In that study, individual rupture stretch and tangent elastic modulus were determined in vitro by means of bulge inflation tests on the identical ATAA wall tissue segments that had been excised intraoperatively. Trabelsi et al. [48] proposed a method for the in vivo identification of the (global) stiffness of an aortic segment based on the determination of volumetric distensibility from gated CT scans and pulse pressure. However, only moderate correlation was found between the global in vivo stiffness values and the (local) stretch based rupture risk criterion determined from bulge inflation tests on excised tissue segments of 13 ATAA. Therefore, the assessment of the local elastic properties of the ATAA-wall from in vivo data that are clinically available, might be a crucial point for establishing a reliable method of estimation of ATAA rupture risk [39]. In the present paper, a new inverse approach to this task is presented.

This is meaningful as stiffening of the aortic wall is both a cause and a consequence of ATAA $[7,50]$. Stiffening leads to a decrease of extensibility due to loss of elastin and deposition of collagen during growth and remodeling. There are studies that have indeed demonstrated such stiffening (without wall weakening) in age-matched subjects $[30,19]$. They mostly found local variations of mechanical parameters along the longitudinal direction, indicating higher strength and peak elastic modulus along the circumferential direction compared to the longitudinal direction [30]. Therefore, identifying non-invasively the in vivo elastic properties of the aorta would help clinicians to take decisions whether the patient needs surgical intervention. Besides, the mechanical properties can be used to perform computational analyses, the accuracy of which will rely on the accuracy of input parameters.

However, non-invasive characterization of elastic properties is not the most common approach for deriving material properties of aortas. The most common methods to obtain material properties are in vitro uniaxial and biaxial mechanical tests $[7,21,40]$. They permit plotting stress-strain curves and deriving parameters 
of strain energy functions or linearized elastic moduli of the aneurysmal walls at different stages of loading [50]. Several studies have characterized the mechanical properties of ATAA via strain energy functions and elastic moduli indicating that ATAA causes stiffening and extensibility reduction of tissue while having no effect on strength reduction. To assess rupture risk on a patient-specific level, their results imply decreased tissue compliance as a rupture risk factor [46,35].

To our best knowledge no study has ever identified regional variations of material properties in ATAAs on a patient-specific basis using a non-invasive methodology. Therefore, the main objective of this work is to present a novel methodology based on gated CT images for the identification of local stiffness properties in ATAAs under assumptions of linearized and isotropic elasticity. After a comprehensive presentation, we show exemplary results on three patients for the proof of concept.

\section{Material and methods}

\subsection{Origin of data}

After informed consent and according to a protocol approved by the Institutional Review Board of the University Hospital Center of Saint-Etienne, non invasive systolic and diastolic blood pressures in brachial artery and gated CT scans were obtained for three patients who underwent elective surgical repair of ATAA at the University Hospital of Saint-Etienne (CHU-SE) in France. The demographic information of these patients is recorded in Table 1. For each patient, images were recorded at ten phases of their cardiac cycle. These images were used to reconstruct the geometry of their whole aorta, including diastolic and systolic phases. Semiautomatic segmentation of the CT image slices was done using MIMICS (v.10.01, Materialise NV). The three-dimensional (3D) surface of the aorta was generated for each phase and exported as STL format. All phases were cut by the same cross sectional planes to predefine a domain of the aorta larger than the final segment of interest in both ascending and descending parts. The purpose was to initiate the procedure in order to run the Vascular Modeling Toolkit (VMTK, Orobix, Bergamo, Italy; www.vmtk.org) [1] and to derive the parametric coordinates of 
85 the surface. Identical smoothing factor was applied at all phases. To recognize the ${ }_{86}$ systolic and diastolic phases, the luminal volumes of all phases were compared. The systolic scan was defined as the one with the largest volume and the diastolic

${ }_{88}$ scan as the one with the smallest volume. See Fig. 1 in which each phase is shown using a different color.

2.2 Theory of the inverse approach

\begin{abstract}
A set of nodes was defined across each reconstructed aortic geometry, with the requirement that a node represented the position of the same material point at each phase of the cardiac cycle. For this, it was essential to reconstruct a structural mesh for all phases with an identical number of elements and nodes and to have a mesh morphing function between the geometries of each phase. The structural mesh was convenient for several reasons; 1- to compare information of each element and nodes in identical position between different phases, 2- to reduce the computational time, 3- structural mesh generally deforms under lower strain energy states.
\end{abstract}

VMTK [1] was employed to generate the structural mesh from STL files. The method was based on centerlines and decomposition of the surface into existing branches and mapping each branch onto template parametric coordinates. Once a bifurcation is split into branches, each branch is topologically equivalent to a cylinder and can be mapped onto a set of rectangular parametric coordinates [1]. The approach for mapping the surface of bifurcations consisted of several steps: calculation of centerlines, definition of the reference system, decomposition of the bifurcation into its branches and their parameterization. VMTK provides a technique based on objective criteria capable of generating consistent parameterizations over a wide range of bifurcating geometries. Let $\partial \Omega_{i}$ be the surface of the $i$ th branch which is delimited by two topological circles $\psi_{i 0}$ and $\psi_{i L}$. Considering $u \in\left[0, L_{i}\right]$ is the longitudinal parametric coordinate and $v \in[-\pi, \pi]$ is the periodic circumferential parametric coordinate, over the parametric space of $U \subset \mathbb{R}^{2}$ in the coordinates $U=(u, v)$, a bijective mapping is derived as [1]

$$
\Phi: \underset{\mathbf{x}}{\partial} \Omega_{i} \rightarrow \underset{\mathbf{u}}{U}
$$


such that $\boldsymbol{\Phi}(\mathbf{x})=(0, v)$ on $\psi_{i 0}$ and $\boldsymbol{\Phi}(\mathbf{x})=\left(L_{i}, v\right)$ on $\psi_{i L}$. Longitudinal mapping in VMTK is created by a harmonic and a stretch function to achieve localization with respect to centerline abcissa and reference system [23]. The harmonic function $f=f(\mathbf{x})$ with $\mathbf{x} \in \partial \Omega_{i}$ is computed by solving the elliptic partial differential equation as

$$
\Delta_{B} f=0
$$

where $\Delta_{B}$ is the Laplace-Beltrami operator. The longitudinal parametric coordinate is expressed by

$$
u(\mathbf{x})=s \circ f(\mathbf{x})
$$

where $s$ is the stretch function and can be defined as

$$
s(f)=\frac{1}{|\lambda(f)|} \int_{\lambda(f)} g(\mathbf{x}) d \lambda
$$

where $\lambda(f)$ indicates a level-set of $f$. The angular position of each node is determined by a set of normals along the curve of frame $\tau$, named $\mathbf{n}(\tau)$ (which is directed towards the center of the osculating circle of the curve at each point), and its nearest point on the centerline, $\mathbf{c}(\tau)$, as

$$
v(\mathbf{x})=\arccos ((\mathbf{x}-\mathbf{c}(\tau)) \cdot \mathbf{n}(\tau))
$$

where $\tau$ indicates the different time frames.

One of the requirements is that the inlets and outlets of the imported mesh into VMTK should be open. Left subclavian artery, brachiocephalic artery, left common carotid artery and coronary artery branches were used to provide anatomical references to ensure that the measurements taken at different phases of the cardiac cycle were at the same location in the aorta (see Fig. 1). Eventually, the extracted data from VMTK were postprocessed in Matlab to extract an accurate mesh using the longitudinal and circumferential metrics obtained from VMTK. A template meshgrid with an arbitrary number of points was created in the $u_{\text {temp }} \in\left[u_{\min }, u_{\max }\right]$ and $v_{\text {temp }} \in[-\pi, \pi]$ domain. A polynomial approximation was implemented around each node of the template meshgrid. For instance, let $\left(u_{\text {temp }}^{i}, v_{\text {temp }}^{i}\right)$ denote the parametric coordinates of node $\mathbf{X}_{i}$. Let $\left(\mathbf{u}_{\mathrm{nb}}, \mathbf{v}_{\mathrm{nb}}\right)$ be the parametric coordinates of the nodes of the reconstructed STL surface around $\mathbf{X}_{i}$. These nodes were selected such that $d u_{j}=\left(u_{\mathrm{nb}}^{j}-u_{\mathrm{temp}}^{i}\right)<\delta_{u}$ 
142 with

and $d v_{j}=\left(v_{\mathrm{nb}}^{j}-v_{\text {temp }}^{i}\right)<\delta_{v}, \delta_{u}$ and $\delta_{v}$ defining the size of the neighborhood. We assumed $\delta_{u}=8 \mathrm{~mm}$ and $\delta_{v}=\pi / 5 \mathrm{rad}$. Therefore using the least-squares method, the polynomial approximation was written such as

$$
\mathbf{A X}=\mathbf{X}_{\mathrm{n} b}
$$

$$
\mathbf{A}=\left[\begin{array}{rrrrrrrr}
1 & d u_{1} & d v_{1} & d u_{1} d v_{1} & d u_{1}{ }^{2} & d v_{1}{ }^{2} & d u_{1}{ }^{2} d v_{1} & d u_{1} d v_{1}{ }^{2} \\
\vdots & \vdots & \vdots & \vdots & \vdots & \vdots & \vdots & \vdots \\
1 & d u_{k} & d v_{k} & d u_{k} d v_{k} & d u_{k}{ }^{2} & d v_{k}{ }^{2} & d u_{k}{ }^{2} d v_{k} & d u_{k} d v_{k}{ }^{2} \\
\vdots & \vdots & \vdots & \vdots & \vdots & \vdots & \vdots & \vdots \\
1 & d u_{n} & d v_{n} & d u_{n} d v_{n} & d u_{n}{ }^{2} & d v_{n}{ }^{2} & d u_{n}{ }^{2} d v_{n} & d u_{n} d v_{n}{ }^{2}
\end{array}\right]
$$

144

$$
\mathbf{X}=\left[\begin{array}{ccc}
X_{1} & Y_{1} & Z_{1} \\
X_{2} & Y_{2} & Z_{2} \\
\vdots & \vdots & \vdots \\
X_{8} & Y_{8} & Z_{8}
\end{array}\right]
$$

and

$$
\mathbf{X}_{\mathrm{nb}}=\left[\begin{array}{ccc}
X_{\mathrm{nb}}^{1} & Y_{\mathrm{nb}}^{1} & Z_{\mathrm{nb}}^{1} \\
\vdots & \vdots & \vdots \\
X_{\mathrm{nb}}^{k} & Y_{\mathrm{nb}}^{k} & Z_{\mathrm{nb}}^{k} \\
\vdots & \vdots & \vdots \\
X_{\mathrm{nb}}^{n} & Y_{\mathrm{nb}}^{n} & Z_{\mathrm{nb}}^{n}
\end{array}\right]
$$

Based on a mesh sensitivity analysis, an average mesh size of $1 \times 1 \mathrm{~mm}$ was used.

According to Wittek et al [52] who observed that the ascending thoracic aorta experiences clockwise and counterclockwise twist, we derived the time variations of the circumferential coordinate, $v$, of coronary arteries in the space of parametric coordinate and subtracted this value from the circumferential coordinate of the assumed reference phase in order to apply torsion during aortic wall motion. To adjust all phases longitudinally, the origin of $u$ was set in the coronary plane at each phase. Accordingly, the torsion was applied by subtracting the shift of 
the $v$ value at the inlet, and by subtracting a shift in the $v$ value all along the thoracic aorta, varying linearly from $v$ to 0 between the inlet and the position of the brachiocephalic artery.

Any periodic function (in this case nodal coordinates of all phases, $x(t)$ ) can be expressed as a weighted sum of infinite series of sine and cosine functions of varying frequency. Therefore, having the structural mesh of all ten phases, a discrete Fourier transform was employed such as

$$
x(t)=a_{0}+\sum_{n=1}^{\infty}\left(a_{n} \cos (n f t)+b_{n} \sin (n f t)\right)
$$

Where $a_{0}$ is the Direct Current (DC) term and the sum is the fundamental magnitude of the time-varying node positions when $n=1$ while $f$ is the fundamental frequency. A strain analysis was performed on the average geometry (defined by using the DC terms of all nodal positions) by applying displacements (including torsion) at each node corresponding to the fundamental term obtained from the Fourier transform in Eq. 10. As the fundamental term is complex, the real and the imaginary parts are applied separately at the corresponding nodes (in their average position, defined by the DC term) using the finite element method (FEM). Eventually the reconstructed strain components were also complex. The components in the longitudinal and circumferential directions were derived respectively as

$$
\begin{aligned}
& \varepsilon_{1}^{\mathrm{com}}=\varepsilon_{1}^{\mathrm{r}}+i \varepsilon_{1}^{\mathrm{im}} \\
& \varepsilon_{2}^{\mathrm{com}}=\varepsilon_{2}^{\mathrm{r}}+i \varepsilon_{2}^{\mathrm{im}}
\end{aligned}
$$

where superscripts " $r$ " and " $i m$ " indicate real and imaginary contributions, respectively. Therefore the magnitude of strains in the longitudinal and circumferential directions can be respectively obtained as

$$
\begin{aligned}
& \varepsilon_{1}=\sqrt{\varepsilon_{1}^{\mathrm{r} 2}+\varepsilon_{1}^{\mathrm{im}^{2}}} \\
& \varepsilon_{2}=\sqrt{\varepsilon_{2}^{\mathrm{r} 2}+\varepsilon_{2}^{\mathrm{im}^{2}}}
\end{aligned}
$$

\footnotetext{
The results of the strain computation are independent of the chosen material properties and this methodology is applicable to finite deformation problems. Although the aortic tissue is globally anisotropic and nonlinear, we linearized here its mechanical behaviour in the range of strains induced by pressure variations between diastole and systole, and neglected anisotropic effects in this range too.
} 
The constitutive equations reduced to Hooke's law in plane stress. Due to the spatial resolution of $\mathrm{CT}$, the local aortic thickness cannot be measured accurately in vivo. The thickness was previously measured in vitro on the excised sample after the surgery [13]. However, the in vitro measurement is performed when the tissue is loadfree, whereas the tissue is stretched and pressurized in vivo. Therefore the thickness can vary significantly in vivo, thus to express the Hooke's law, we wrote the membrane tensions, $\tau$, as

$$
\begin{aligned}
& \tau_{1}(t)=\tau_{1}^{0}+Q\left(\varepsilon_{1}(t)+\nu \varepsilon_{2}(t)\right) \\
& \tau_{2}(t)=\tau_{2}^{0}+Q\left(\nu \varepsilon_{1}(t)+\varepsilon_{2}(t)\right)
\end{aligned}
$$

where $\tau_{1}^{0}$ and $\tau_{2}^{0}$ are the DC terms of both components of the membrane tension (representing pretensions existing in the average geometry due to average blood pressure and axial tension), $Q$ is the membrane stiffness and $\nu$ is the Poisson's ratio in which incompressibility is assumed $(\nu=0.49)$. We derived $\tau_{1}^{0}$ and $\tau_{2}^{0}$ by achieving an FE stress analysis on the average geometry under the effect of the average pressure $P_{0}$ using the approach proposed by [32].

As shown in Fig. 2 it is assumed that each element of the arterial wall is a finite sector of an ellipsoidal membrane with radii $r_{1}$ and $r_{2}$ in both circumferential and longitudinal directions. So, in the framework of these assumptions, local equilibrium equations can be written as

$$
P(t)=\frac{\tau_{1}(t)}{r_{1}(t)}+\frac{\tau_{2}(t)}{r_{2}(t)}
$$

where $r_{1}$ and $r_{2}$ are the radii of the element sector in both directions (major and minor) as shown in Fig. 2 and $P$ is the applied pressure. Using $P_{0}$ to denote the DC term of pressure variations, and neglecting second order variations, Eq. 14 can be rewritten:

$$
\begin{aligned}
& P(t)-P_{0}=\frac{\tau_{1}(t)-\tau_{1}^{0}}{r_{1}^{0}}+ \\
& \frac{\tau_{2}(t)-\tau_{2}^{0}}{r_{2}^{0}}-\frac{\tau_{1}^{0}\left(r_{1}(t)-r_{1}^{0}\right)}{\left(r_{1}^{0}\right)^{2}}-\frac{\tau_{2}^{0}\left(r_{2}(t)-r_{2}^{0}\right)}{\left(r_{2}^{0}\right)^{2}}
\end{aligned}
$$

Using $\Delta P, \Delta \tau_{1}, \Delta \tau_{2} \Delta r_{1}$ and $\Delta r_{2}$ to denote the fundamentals of pressure, the circumferential and longitudinal components of the local tension tensor, and the circumferential and longitudinal radius of curvature, respectively, Eq. 15 can be written as follows: 


$$
\Delta P=\frac{\Delta \tau_{1}}{r_{1}^{0}}+\frac{\Delta \tau_{2}}{r_{2}^{0}}-\frac{\tau_{1}^{0}\left(\Delta r_{1}\right)}{\left(r_{1}^{0}\right)^{2}}-\frac{\tau_{2}^{0}\left(\Delta r_{2}\right)}{\left(r_{2}^{0}\right)^{2}}
$$

In the following, we assume $\Delta P=\left(P_{\text {sys }}-P_{\text {dias }}\right) / 2$.

Substituting Eqs. 13 in 16, we obtain the membrane stiffness for each element such as

$$
Q=\frac{\Delta P+\frac{\tau_{1}^{0} \Delta r_{1}}{\left(r_{1}^{0}\right)^{2}}+\frac{\tau_{2}^{0} \Delta r_{2}}{\left(r_{2}^{0}\right)^{2}}}{\frac{\varepsilon_{1}(t)+\nu \varepsilon_{2}(t)}{r_{1}^{0}}+\frac{\nu \varepsilon_{1}(t)+\varepsilon_{2}(t)}{r_{2}^{0}}}
$$

To obtain $r_{1}$ and $r_{2}$ fast and efficiently, we developed a method based on the principle of virtual work as previously introduced in [4], so assuming stresses $\sigma$ at equilibrium with external loads $\mathbf{T}$ should satisfy the following equation:

$$
\int_{v} \sigma i j: \varepsilon_{i j}^{*} d \nu+\int_{\partial \nu} T_{i} u_{i}^{*} d s=0
$$

Where $v$ and $s$ are the volume and surface of the domain of interest, respectively, $\mathbf{u}^{*}$ is a virtual continuous displacement field and $\varepsilon^{*}$ are the virtual strains related to the gradients of the virtual displacements. As this equation is valid for any virtual field, $\mathbf{u}^{*}$ can be defined as a unit vector oriented along the normal directions of the aortic wall at every node (directions $n_{1,2,3,4}^{n}$ in Fig. 2). Accordingly, it is possible to derive the following local equation:

$$
\sigma_{1} h \varepsilon_{1}^{*}+\sigma_{2} h \varepsilon_{2}^{*}=p u^{*}
$$

Where $\sigma_{1}$ and $\sigma_{2}$ are the principal components of the stress and $h$ is the thickness. The Laplace law may be written:

$$
\frac{\sigma_{1}}{r_{1}} h+\frac{\sigma_{2}}{r_{2}} h=p
$$

By identification between the two previous equations, we obtain directly a relationship between the local radii of curvature and the virtual strain fields. We applied a displacement with arbitrary magnitude of $u^{*}$ in the normal directions to each node (directions $n_{1,2,3,4}^{n}$ in Fig. 2) to inflate virtually the aorta. After recording $\varepsilon_{1}^{*}$ and $\varepsilon_{2}^{*}$ in the longitudinal and circumferential directions induced by this virtual inflation, the radii can be calculated as

$$
\begin{aligned}
\frac{1}{r_{1}} & =\frac{\varepsilon_{1}^{*}}{u^{*}} \\
\frac{1}{r_{2}} & =\frac{\varepsilon_{2}^{*}}{u^{*}}
\end{aligned}
$$


In this work we introduce the concept of "extensional stiffness" which equals the material stiffness times the thickness and whose dimension is MPa.mm.

Stiffness variations across the different regions of the thoracic aorta may exist at different scales. In order to filter out high frequency variations, the obtained results were eventually fitted using Fourier polynomial of order 4 along the circumferential directions and polynomials of order 3 along the axial direction, permitting a smoother estimation of stiffness distribution.

\subsection{Numerical implementation}

In the current work we reconstructed ascending and descending aortas excluding all branches. Therefore, the right coronary and brachiocephalic arteries were used as reference points to obtain identical parts of the ascending aorta at different phases and the left subclavian artery was used as a reference point to consider identical parts of the descending aorta at different phases. For each patient and each phase, a structural mesh using quadrilateral elements with four nodes was prepared and then the average structural meshes of ascending and descending aortas were obtained by averaging the nodal positions throughout the cardiac cycle. Afterwards, for each aorta, the Abaqus FE software [24] was employed to calculate $\varepsilon_{1}^{*}$ and $\varepsilon_{2}^{*}$ by applying $u^{*}$ at each node in normal directions of $n_{1}^{n}: n_{4}^{n}$ in Fig. 2. Moreover, two other independent FE analyses were performed by applying corresponding real and imaginary displacements at each node (including aorta torsion) to calculate $\varepsilon_{1}^{\text {com }}$ and $\varepsilon_{2}^{\text {com }}$ and subsequently $\varepsilon_{1}$ and $\varepsilon_{2}$ expressed in Eq. 12. For each FE analysis, orientation user subroutine (ORIENT) was employed to assign local material directions in order to save results in the local coordinate system. Each geometry was a non-perfectly cylindrical geometry so that the radial direction (normal to the artery) was defined as the outward normal direction to each element, the axial direction was defined as the direction parallel to the luminal centerline in the direction of the blood flow, and the circumferential direction was perpendicular to both previously defined directions. To calculate and visualize the local membrane stiffness expressed in Eq. 17, a user material subroutine (UMAT) and a user-defined external databases (UEXTERNALDB) were coupled with the 
commercial FE software Abaqus [24]. The complete methodology is summarized in a flowchart shown in Fig. 3 .

In this work, we had to perform several different steps to characterize the local aortic stiffness for each patient. The most time consuming step was the segmentation of CT images (cleaning could last one day for the noisiest). Generation of the structural mesh took about 10 minutes including VMTK and further processing. It took less than 2 hours to prepare the ABAQUS input files for stress analyses, strain analyses, stiffness assessment and reconstruction. Each ABAQUS resolution took about five minutes.

\section{Results}

\subsection{Numerical validation}

Numerical validation was performed considering two different material behaviors; linear elastic and non-Linear anisotropic material properties.

\subsection{Linear elastic material property}

For the sake of validation, we used a reference aortic geometry (RG) and we created numerically a deformed aortic geometry (DG) by considering the effects of an average intramural pressure $(\mathrm{P}=40 \mathrm{mmHg}(5.33 \mathrm{KPa}))$ onto the aorta modeled with a linear elastic behavior. The DG was derived by a finite element analysis using the raw STL mesh of the reference geometry. Two analyses (cases) were performed: one with a homogeneous stiffness in the whole aorta and another one with a heterogeneous stiffness distribution in which three regions were defined in the aorta, each one with different stiffness shown in Fig. 6. Each analysis provided a deformed STL mesh. Displacement maps used to reconstruct DG are shown in Fig. 4. Having in hand RG and DG for both cases, we performed our approach using VMTK and MATLAB to independently generate the structural mesh of each geometry. For the sake of validation, we subtracted the nodal coordinates 
of both structural meshes (on RG and DG). As it can be observed in Fig. 5, we found a good agreement between the displacements reconstructed using our approach and the reference displacement, indicating that structural meshes of both geometries are related to nearly similar material points. After this first validation on the reconstructed displacement data, we applied the whole methodology with reconstruction of stiffness distributions from the two simulated cases. For each case, a stiffness distribution was obtained and compared to the reference one (Fig. 6). The reconstructed stiffness was in good agreement with the reference and especially the local heterogeneity could be well retrieved for the heterogeneous case. Artifacts only appear very locally at the proximal and distal parts of the aortic segment due to spurious effects of polynomial smoothing close to the boundary (edge effects).

\subsection{Non-linear anisotropic material property}

We have performed a complementary validation of our methodology considering anisotropy and effects of nonlinearity. We used the same aortic geometry (raw stl) as the one used for the linearized case study, which is the aortic geometry of a real patient which was segmented from a CT scan obtained at $P_{\text {diastole }}=80$ mmHg $(10.66 \mathrm{KPa})$. We modeled the aortic wall with a Gasser-Ogden-Holzapfel anisotropic strain energy function which may be written as [26]

$$
\bar{\Psi}=C_{10}\left(\bar{I}_{1}-3\right)+\frac{k_{1}}{2 k_{2}} \sum_{i=4,6}\left[\exp \left[k_{2}\left(\bar{I}_{i}-1\right)^{2}\right]-1\right]+\kappa(J-1)^{2}
$$

where $C_{10}$ and $k_{1}$ are material parameters and have a stress-like dimension, and $k_{2}$ is a dimensionless material parameters while $\kappa$ is bulk modulus and $J$ is Jacobian (local volume ratio.). $\bar{I}_{1}=\operatorname{tr}(\bar{C}), \bar{I}_{4}=\bar{C}: a_{01} \otimes a_{01}$ and $\bar{I}_{6}=\bar{C}: a_{02} \otimes a_{02}$ are first, fourth and sixth invariants of the modified counterparts of the right CauchyGreen tensor, $\bar{C}$. Note that the families of collagenous fibers are characterized by the two reference direction vectors $a_{0 j}=\left[0 \cos \theta_{j} \sin \theta_{j}\right], j=1,2$, with $\left|a_{0 j}\right|=1$. $\theta_{j}$ is the angles between the collagen fibers (arranged in symmetrical spirals) and the circumferential direction.

Two analyses were performed: One with a homogeneous distribution of hyperelastic material properties in the whole the aorta and the other with a heterogeneous distribution of hyperelastic material properties, considering three regions. Material 
parameters used for this validation study are reported in Table 2. Before applying these two models to calculate displacement between diastole and systole, we first computed the zero pressure geometry in each case using the approach presented by Mousavi et al $[38,39]$. Afterwards, we calculate the deformation of the aortic geometry for a systolic pressure directly by applying $P_{\text {systole }}=120 \mathrm{mmHg}(16 \mathrm{KPa})$ onto the zero pressure geometry.

Having in hand the diastolic geometry and the computed systolic geometry, we ran our approach using VMTK and MATLAB to independently generate the structural mesh of each geometry (systolic and diastolic) in both homogeneous and heterogeneous analysis. We subtracted the nodal coordinates of both structural meshes (systolic and diastolic phases) to obtain the displacements between systole and diastole for each case. Assuming the diastolic phase as the reference geometry we applied our methodology to retrieve the stiffness of aorta. The identified results shown in Fig. 7 demonstrate that our methodology is able to retrieve the "stiff" region in the heterogeneous case, whereas the homogeneous case show only small variations which are mostly related to edge effects. However, this remains a qualitative comparison as the obtained linearized stiffness is not directly related to hyperelastic properties. This qualitative validation completes well the previous quantitative validation where we modeled the aorta with a linear elastic behavior and where we were able to retrieve the correct stiffness properties.

\subsection{Patient-specific results}

The methodology described previously was applied on 3 patients. The membrane stiffness of their aorta was reconstructed at every Gauss point of the mesh (excluding the branches and the region of the arch between the brachiocepohalic and the left subclavian arteries) and then the values were interpolated at nodal positions. The results were plotted as independent colormaps which are shown in Fig. 8. The distributions appear heterogeneous, especially in the ATAA regions where some regions of relatively higher membrane stiffness appear. The distribution of local membrane stiffness showed higher values for ascending aortas compared to descending aortas. 
3.5 Comparison of the results

For these 3 patients, elastic properties of their ATAA were previously characterized with two other methods:

1. An ATAA segment was defined by two transverse planes located respectively at $10 \mathrm{~cm}$ and $55 \mathrm{~cm}$ from the coronary artery. Across the defined segment, the average distensibility of the ascending thoracic aorta was assessed from CT scans according to [48]

$$
D_{V}=\frac{\Delta V}{V \Delta P}
$$

Where $V, \Delta V$ and $\Delta P$ are respectively the luminal volume, the volume difference between diastole and systole and the pressure difference. The membrane stiffness was deduced as the ratio between the distensibility and the average diameter of the segment. Let $E_{\text {in-vivo }}$ denote the membrane stiffness obtained with this method.

2. After the surgical intervention on these patients, an ATAA segment was excised. From this segment, a square $(45 \times 45 \mathrm{~mm})$ was cut in the outer curvature region and tested in bulge inflation. The stress-strain response of the tested sample was linearized over a range of pressures inducing similar tensions in the tissue as the tensions produced by diastolic and systolic pressures. The obtained linearized stiffness of the tissue over this range, multiplied by tissue thickness, yielded another estimate of the membrane stiffness, which was denoted $E_{\text {in-vitro }}$.

Therefore, for each patient, we focused on the distribution of membrane stiffness across the ATAA segment and across the cut square (Fig. 9). For the sake of statistical analysis, we derived the median and interquartile ranges of all nodal values of the identified membrane stiffness (Fig. 10). The analysis was achieved first by taking all the values of the ascending aorta, then only the values in the ATAA segment and finally only the values of the cut square. For each patient, we also plotted in Fig. 10 the $E_{\text {in-vitro }}$ and $E_{i n-v i v o}$ values obtained by [13] and [48], respectively. This analysis showed that the median and interquartile values for different cuts of all patients are relatively at the same ranges. Comparing the interquartile box obtained in this study with the corresponding results of [48], 
it can be deduced that for all patients $E_{\text {in-vivo }}$ is a value between the lower and the median value (mostly close to median) while $E_{i n-v i t r o}$ values are close to lower corresponding interquartile. This can be explained by the mechanical actions of surrounding tissues and environment in vivo, which may apply a counter pressure on the artery. We did not take into account this counter pressure in the identification as $P(t)$ in Eq. 14 .

\section{Discussion}

In this paper we have introduced an original method to reconstruct the stiffness distribution of the thoracic aorta from gated CT images considering ten phases of a cardiac cycle. This methodology requires tracking the deformations of the aortic wall throughout a cardiac cycle from the gated CT images. The deformations were tracked without any additional information or marker indicating heterogeneous deformation in between the bifurcations.

\subsection{General remarks}

For the first time ever, regional variations of stiffness properties across ATAA were reconstructed on 3 patients non-invasively. To achieve this reconstruction, a novel non-invasive inverse method was developed based on preoperative gated CT scans. The non invasive identification of elastic properties of ATAA is highly important as our research group $[13,48]$ recently showed that the membrane stiffness is significantly correlated with the stretch based rupture risk of ATAA. Therefore, it is significantly interesting to obtain the local membrane stiffness of the ascending thoracic aorta to detect pathological regions with the highest risks of rupture. Interestingly, our results indicate that the local membrane stiffness is higher in the aneurysmal ascending thoracic aorta than in the descending aorta, and that the values are relatively higher than stiffness properties reported for healthy aortas. However, despite this general trend, the distribution of membrane stiffness is heterogeneous in the aneurysmal region, with large stiffness in the bulging part of aneurysm. The local loss of elasticity can be related to a more pronounced local remodeling with, for instance, a larger fraction of collagen fibers. Sassani et al [43] 
showed a large distribution of fiber orientations spanning from circumferential to longitudinal directions, which was also supported by [46] stating that ATAA had no effect on strength. However, this can cause stiffening and extensibility reduction, corroborated with histological observation of elastin degradation but not collagen content. It could be interesting also to check the possible correlation of these regions with local concentration of wall shear stress induced by the blood flow [34]. All these possible correlations between regional variations of membrane stiffness and local tissue composition or local hemodynamics effects are currently under study in our group on a cohort of patients. Over the past decades the biomechanical properties of the aorta have been widely investigated $[7,50,21,40,18,12,25$, $37,41,44]$. As the aortic tissues are intrinsically anisotropic, the identified stiffness provided by our method combines effects of the axial and circumferential extensional linearized stiffness. However, as we use only the pressure loading to solve the identification problem (we have no information on the axial tension in vivo), it is dominated by effects of the circumferential extensional linearized stiffness. This is in agreement with conclusions of Ferrara et al. [16] who indicated that the weight of the circumferential elastic modulus is significantly higher than the weight of the longitudinal one in the aortic mechanical response to pressure variations, while there was no significant difference in the elastic modulus between anterior and posterior regions in the longitudinal direction. The maximum circumferential elastic modulus was reported in posterior region. Moreover, their results indicate that regardless of age and gender, the mean elastic modulus is larger in the circumferential direction. However aging reduces the mean elastic modulus and male has higher mean elastic modulus in comparison with female. Iliopoulos et al [30] support these results and state that circumferential specimens were stiffer than longitudinal ones in the posterior, left lateral, and all regions at physiologic and high stresses but not at low stresses. Furthermore, in agreement with Ferrara et al. [16] they found no significant regional variations of aortic stiffness in the circumferential direction, and similarly at low stresses for longitudinal specimens, while at physiologic and high stresses the right lateral was the stiffest ATAA region. In the other study by the same group [29] it was shown that peak elastic modulus in the anterior region was significantly lower than that in the right, but not the left, lateral and posterior regions. Choudhury et al [7] studied local mechanical 
properties of healthy and diseased, bicuspid and tricuspid aortic valves (BAV and TAV), human ascending aorta. Indicating that BAV tissue was the thinnest and contained the largest fraction of collagen, they found significantly less elastin both in TAV and BAV tissue in contrast to healthy tissue, while on average the smallest stiffness was observed in TAV patients. The elastic modulus was not dependent on direction (isotropic) but it was dependent on the quadrant and tissue type. The medial quadrant was the most compliant and the lateral quadrant tissue was the stiffest.

Although most of these previous investigators carried out experimental tests on tissue samples excised from patients during surgery, or on cadavers, the material properties of tissues are commonly characterized averagedly across these samples and in conditions which may be far from in vivo conditions, such as for instance tensile test. Only recently, the use of digital image correlation (DIC) has made it possible to investigate the local material properties of excised aortic samples $[2$, $11,10]$. Inverse methods were developed to process DIC measurements and reconstruct the regional variations of material properties, as for instance using a tension inflation tests keeping the cylindrical shape of the aorta [4]. Regional variations of constitutive properties make sense as they can be related to local tissue adaptation through growth and remodeling [28].

When excised aortic samples are tested in vitro, the results usually take the form of constitutive parameters in a strain energy density function [27]. Strain energy density functions usually have a large number of constitutive parameters with possible couplings between each other and it was recently suggested to evaluate more functional parameters, such as for instance the linearized in vivo stiffness [4]. Here, we focused only on this linearized in vivo stiffness as we had only a non invasive loading of the aorta across a limited range of strains through the variations of blood pressure. The non invasive identification of the aortic stiffness was previously achieved averagedly across aortic segments either by measuring the aortic distensibility [48] or by measuring the pulse wave velocity [22], and relating these quantities to the aortic stiffness. Local non invasive identification was previoulsy achieved using $4 \mathrm{D}$ ultrasound on the abdominal aorta $[32,51]$ but the present study is the first one using gated $\mathrm{CT}$ scans and applying the approach onto the ascending thoracic aorta. The availability of these images allowed us 
to model subject-specific arterial configurations. Since a correlation between the stretch-based rupture risk and the tangent elastic modulus was found by [13], the methodology proposed here has potential to identify the rupture risk of aorta according to calculated local membrane stiffness.

It is important to point out the benefit of using a Fourier analysis (as done here for the regional reconstruction) instead of using simply diastolic and systolic geometries of the aorta (as done for distensibility measurements). Indeed, the distensibility is usually assessed by measuring the change of cross section area of the aorta between diastole and systole, diastole being defined as the stage with minimum cross section area and systole being defined as the stage with maximum cross section area. There is always a significant uncertainty on the times at which the 10 phases of CT scans are acquired. If we could reduce this uncertainty and provided that a pressure curve can be measured simultaneously, the method could be easily extended to derive time variations of the stiffness throughout the whole cardiac cycle. However, we noticed that locally, the maximum deflection of the aorta, and its maximum strains, are not reached at the same stage of the cardiac cycle depending on the position (Fig. 1). It means that the response of the aorta to the pressure variations presents phase shifts depending on the position. These phase shifts are potentially the result of viscous effect but also of the combined action of the blood pressure and of the cardiac motion, which are not synchronized, as this was previously shown by [52]. Due to these local phase shifts, it happens that the maximum strain of the aorta is not reached at systole for all the positions and the minimum strain of the aorta is not reached at diastole. Therefore, taking the average change of strain between diastole and systole to derive the aortic stiffness, as done in the distensibility method [13,48], automatically underestimates the actual strain variations occurring throughout a cardiac cycle. The consequence of this strain underestimation should be a stiffness overestimation for the distensibility method. However, in this work, it can be compensated by another effect which we would like to point out here as well. Commonly, pressure variations are linearly related to stress variations without considering the effects of radius variations as we did in Eq. 16. However, we found that the term considering the effects of radius variations, namely $\frac{\tau_{1}^{0}\left(\Delta r_{1}\right)}{\left(r_{1}^{0}\right)^{2}}-\frac{\tau_{2}^{0}\left(\Delta r_{2}\right)}{\left(r_{2}^{0}\right)^{2}}$, is averagely responsible for about $10 \%$ of $\Delta P$, with local effects possibly reaching $50 \%$. Neglecting this term, as it is tra- 
ditionally done in distensibility assessment, induces an underestimation of stress variations. Combined with the strain underestimation explained previously, the stress underestimation results in a good agreement of the distensibility method with our novel inverse method. Nevertheless, future stiffness identifications should take into account all these effects as done in the current study.

\subsection{Limitations}

There are several limitations in this work which can be discussed as below:

- The inverse approach is based on local equilibrium equations similar to the ones derived in [4]. These equations are obtained based on the principle of virtual work. It is worth noting that they are equivalent to the generalized Laplace's law which writes the local equilibrium between pressures and tensions in a membrane. Accordingly, it means that it is assumed that the aortic wall behaves as a membrane with no bending moments or no through thickness shear. Regions near the branches may not satisfy the membrane assumption, and were removed from the analysis.

- The present study used gated CT scans as a source of dynamic images of the aortic wall during the cardiac cycle. The use of Xray may present a limitation for an extensive use of the methodology, especially if one wants to repeat the identification of aortic stiffness at different ages of ATAA growth, which would induce repeated irradiation for the patient. We are currently working on an extension of the method to images acquired with $4 \mathrm{D}$ MRI.

- The wall is assumed as a linearly elastic material within the systolic-diastolic range and we disregarded the layer-specific wall properties and residual stresses existing in the ATAA wall.

- We here assumed an isotropic behavior of the arterial wall. It was not possible to consider anisotropic effects as only one type of loading was available: variations of the blood pressure. The characterization of anisotropic materials requires loading samples in different directions. The consequence of the isotropy assumption in our approach is that the identified membrane stiffness is a combination of the axial and circumferential stiffness, which may be different. However, the local curvatures being involved in Eq. 17, most of the 
weight is put on circumferential effects (the circumferential radius of curvature being significantly lower than the axial one), meaning that the identified membrane stiffness would be closer to the circumferential stiffness than to the axial stiffness if there was a significant different between both.

- Average tensions existing in the aorta due to the action of the average blood pressure are also involved in Eq. 17. This comes from geometrical nonlinearity involved in finite deformations [3]. These tensions were approximated using the approach of [32]. This approach consists in achieving a linear elastic stress analysis on the same structure as the aorta, assigning a very large elastic modulus. The approach is valid for membrane structures. The membrane assumption was previously shown to be a good approximation for elastostatics of aortic aneurysms [33].

- Due to the removal of in vivo loadings, the artery experiences an elastic recoiling. Even when these loadings are removed, internal or residual stresses still exist in the tissues. An effect of these internal stresses can be observed in arteries with the well-known opening angle [38]. Moreover, Sokolis et al [45] have provided evidence of residual stresses in both the circumferential and longitudinal directions considering different wall layers of ATAA. Therefore, disregarding the presence of residual stresses and considering the arterial wall as a 3D membrane with uniform thickness can be considered as additional limitations of the current work. Nevertheless, despite these limitations, the structural membrane stiffness provided by our methodology is still a useful indicator of local tissue altered remodeling.

- The segmented geometries have some local irregularities (bumps) due to image noise at each phase of the cardiac cycle. The spatial frequency of these irregularities is high and their effect is filtered out by Fourier polynomial smoothing. However, if one wanted to obtain spatial variations of material properties with a higher spatial resolution, one would have to address the issue of these geometric irregularities. 


\section{Conclusion}

Regional variations of stiffness properties across ATAA were reconstructed on 3 patients non invasively. To achieve this reconstruction, a novel non-invasive inverse method was developed based on preoperative gated CT scans. The non invasive identification of elastic properties of ATAA is highly important as our research group $[13,48]$ recently showed that the membrane stiffness is significantly correlated with stretch based rupture risk of ATAA. It would be interesting in future studies to consider a cohort with a larger number of patients in order to interpret regional variations of stiffness properties in ATAAs in terms of local hemodynamics effects and of local tissue remodeling.

\section{Acknowledgements}

The authors are grateful to the European Research Council for grant ERC-2014CoG BIOLOCHANICS.

\section{Conflict of interest}

The authors have no conflicts of interest to declare concerning the contents of this manuscript.

\section{References}

1. L. Antiga and D.A. Steinman. Robust and objective decomposition and mapping of bifurcating vessels. IEEE Trans Med Imaging, 23(6):704-13, 2004.

2. S. Avril, P. Badel, and A. Duprey. Anisotropic and hyperelastic identification of in vitro human arteries from full-field optical measurements. Journal of biomechanics, 43:2978-85, 2010 .

3. S. Baek, R.L. Gleason, K.R. Rajagopal, and J.D. Humphrey. Theory of small on large: potential utility in computations of fluid-solid interactions in arteries. Comput Methods Appl Mech Eng, 196:3070-78, 2007.

4. MR. Bersi, C. Belliniand, P. Di Achille, J.D. Humphrey, K. Genovese, and S. Avril. Novel methodology for characterizing regional variations in the material properties of murine aortas. Journal of biomechanical engineering, 138:071005, 2016. 
5. L.K. Bickerstaff, P.C. Pairolero, L.H. Hollier, L..J. Melton, H.J. Van Peenen, and K.J. Cherry et al. Thoracic aortic aneurysms: a population-based study. Surgery, 92:110308, 1982 .

6. K.H. Chau and J.A. Elefteriades. Natural history of thoracic aortic aneurysms: size matters and plus moving beyond size. Prog Cardiovasc Dis, 56(1):74-80, 2013.

7. N. Choudhury, B. Olivier, R. Leonie, T. Dominique, C. Raymond, and B. Jagdish et al. Local mechanical and structural properties of healthy and diseased human ascending aorta tissue. Cardiovasc Pathol, 18:83-91, 2009.

8. M.A. Coady, J.A. Rizzo, G.L. Hammond, G.S. Kopf, and J.A. Elefteriades. Surgical intervention criteria for thoracic aortic aneurysms: a study of growth rates and complications. Ann Thorac Surg, 67(6):1922-6, 1999.

9. M.A. Coady, J.A. Rizzo, G.L. Hammond, D. Mandapati, U. Darr, G.S. Kopf, and J.A. Elefteriades. What is the appropriate size criterion for resection of thoracic aortic aneurysms? $J$ Thorac Cardiovasc Surg, 113(3):476-91, 1997.

10. F.M. Davis, Y. Luo, S. Avril, Ambroise A. Duprey, and J. Lu. Local mechanical properties of human ascending thoracic aneurysms. Journal of the mechanical behavior of biomedical materials, 61:235-49, 2016.

11. FM. Davis, Y. Luo, S. Avril, A. Duprey, and J. Lu. Pointwise characterization of the elastic properties of planar soft tissues: application to ascending thoracic aneurysms. Biomechanics and modeling in mechanobiology, 14:967-78, 2015.

12. A. Duprey, K. Khanafer, M. Schlicht, S. Avril, D. Williams, and R. Berguer. In vitro characterisation of physiological and maximum elastic modulus of ascending thoracic aortic aneurysms using uniaxial tensile testing. Eur J Vasc Endovasc Surg, 39(6):700-7, 2010.

13. A. Duprey, O. Trabelsi, M. Vola, J.P. Favre, and S. Avril. Biaxial rupture properties of ascending thoracic aortic aneurysms. Acta Biomater, 42:273-85, 2016.

14. T. McGloughlin (Ed.), editor. Biomechanics and mechanobiology of aneurysms. SpringerVerlag, Heidelberg, 2011.

15. J.A. Elefteriades and E.A. Farkas. Thoracic aortic aneurysm clinically pertinent controversies and uncertainties. J Am Coll Cardiol, 55(9):841-57, 2010.

16. A. Ferrara, S. Morganti, P. Totaro, A. Mazzola A, and F. Auricchio. Human dilated ascending aorta: Mechanical characterization via uniaxial tensile tests. J Mech Behav Biomed Mater, 53:257-71, 2016.

17. M.F. Fillinger, S.P. Marra, M.L. Raghavan, and F.E. Kennedy. Prediction of rupture risk in abdominal aortic aneurysm during observation: wall stress versus diameter. $J$ Vasc Surg, 37(4):724-32, 2003.

18. Y.C. Fung. Biomechanics. Mechanical properties of living tissues. Springer, 1993.

19. C.M. García-Herrera, J.M. Atienza, F.J. Rojo, E. Claes, G.V. Guinea, D.J. Celentano, C. García-Montero, and R.L. Burgos. Mechanical behaviour and rupture of normal and pathological human ascending aortic wall. Med Biol Eng Comput, 50(6):559-66, 2008. 
20. J.P. Vande Geest, E.S. Di Martino, A. Bohra, M.S. Makaroun, and D.A. Vorp. A biomechanics-based rupture potential index for abdominal aortic aneurysm risk assessment: demonstrative applicatio. Ann N Y Acad Sci, 1085:11-21, 2006.

21. J.P. Vande Geest, M.S. Sacks, and D.A. Vorp. Age dependency of the biaxial biomechanical behavior of human abdominal aorta. J Biomech Eng, 126:815-22, 2004.

22. M. Groenink, A. de Roos, BJ. Mulder, JA. Spaan, and EE. van der Wall. Changes in aortic distensibility and pulse wave velocity assessed with magnetic resonance imaging following beta-blocker therapy in the marfan syndrome. Am J Cardiol, 82:203-8, 1998.

23. S. Haker, S. Angenent, A. Tannenbaum, and R. Kikinis. Nondistorting flatting maps and the 3-D visualization of colon ct images. IEEE trans. med. imaging, 19:665-670, 2000.

24. Hibbit, Karlson, and Sorensen. Abaqus-Theory manual, 6.11-3 edition, 2011.

25. G.A. Holzapfel. Determination of material models for arterial walls from uniaxial extension tests and histological structure. J Theor Biol, 238:290-302, 2006.

26. GA. Holzapfel, TC. Gasser, and RW. Ogden. A new constitutive framework for arterial wall mechanics and a comparative study of material models. Journal of elasticity and the physical science of solids, 61 (1):1-48, 2000.

27. G.A. Holzapfel, T.C. Gasser, and R.W. Ogden. A new constitutive framework for arterial wall mechanics and a comparative study of material models. Journal of elasticity and the physical science of solids, 61(1-3):1-48, 2000.

28. J.D. Humphrey. Vascular adaptation and mechanical homeostasis at tissue, cellular, and sub-cellular levels. Cell Biochem Biophys, 50:53-78, 2008.

29. D.C. Iliopoulos, R.P. Deveja, E.P. Kritharis, D. Perrea, G.D. Sionis, K. Toutouzas, C. Stefanadis, and D.P. Sokolis. Regional and directional variations in the mechanical properties of ascending thoracic aortic aneurysms. Med Eng Phys, 31(1):1-9, 2008.

30. D.C. Iliopoulos, E.P. Kritharis, A.T. Giagini, and D.P. Sokolis S.A. Papadodima. Ascending thoracic aortic aneurysms are associated with compositional remodeling and vessel stiffening but not weakening in age-matched subjects. J Thorac Cardiovasc Surg, 137(1):101-9, 2009.

31. G. Johansson, U. Markström, and J. Swedenborg. Ruptured thoracic aortic aneurysms: a study of incidence and mortality rates. J Vasc Surg, 21(6):985-8, 1995.

32. G.R. Joldes, K. Miller, A. Wittek, and B. Doyle. A simple, effective and clinically applicable method to compute abdominal aortic aneurysm wall stress. J Mech Behav Biomed Mater, 58:139-48, 2016.

33. J. Lu, X. Zhou, and M.L. Raghavan. Inverse elastostatic stress analysis in pre-deformed biological structures: demonstration using abdominal aortic aneurysms. J Biomech, 40:693$6,2007$.

34. P.G. Malvindi, S. Pasta, G.M. Raffa, and S. Livesey. Computational fluid dynamics of the ascending aorta before the onset of type a aortic dissection. European Journal of Cardio-Thoracic Surgery, 51(3):597-99, 2016.

35. C. Martin, W. Sun, T. Pham, and J. Elefteriades. Predictive biomechanical analysis of ascending aortic aneurysm rupture potential. Acta Biomater, 9(12):9392-400, 2013. 
36. G. Martufi, T.C. Gasser, J.J. Appoo, and E.S. Di Martino. Mechano-biology in the thoracic aortic aneurysm: a review and case study. Biomechanics and modeling in mechanobiology, 13:917-928, 2014.

37. D. Mohan and J.W. Melvin. Failure properties of passive human aortic tissue. I-uniaxial tension tests. J Biomech, 15:887-902, 1982.

38. S.J. Mousavi and S. Avril. Patient-specific stress analyses in the ascending thoracic aorta using a finite-element implementation of the constrained mixture theory. Biomech Model Mechanobiol, 16(5):1765-77, 2017.

39. S.J. Mousavi, S. Farzaneh, and S. Avril. Computational predictions of damage propagation preceding dissection of ascending thoracic aortic aneurysms. Int $J$ Numer Method Biomed Eng, 34(4):e2944, 2017.

40. R.J. Okamoto, J.E. Wagenseil, W.R. DeLong, S.J. Peterson, N.T. Kouchoukos, and T.M. Sundt 3rd. Mechanical properties of dilated human ascending aorta. Ann Biomed Eng, 30:624-35, 2002.

41. P.J. Prendergast, C. Lally, S. Daly, A.J. Reid, T.C. Lee, and D. Quinn et al. Analysis of prolapse in cardiovascular stents: a constitutive equation for vascular tissue and finiteelement modelling. J Biomech Eng, 125:692-99, 2003.

42. V.S. Ramanath, J.K. Oh, T.M. Sundt 3rd, and K.A. Eagle. Acute aortic syndromes and thoracic aortic aneurysm. Mayo Clin Proc, 84(5):465-81, 2009.

43. S.G. Sassani, S. Tsangaris, and D.P. Sokolis. Layer- and region-specific material characterization of ascending thoracic aortic aneurysms by microstructure-based models. $J$ Biomech, 48(14):3757-65, 2015.

44. D.P. Sokolis. Passive mechanical properties and structure of the aorta: segmental analysis. Acta Physiol (Oxf), 190:277-289, 2007.

45. D.P. Sokolis. Effects of aneurysm on the directional, regional, and layer distribution of residual strains in ascending thoracic aorta. J Mech Behav Biomed Mater, 46:229-43, 2015 .

46. D.P. Sokolis, E.P. Kritharis, A.T. Giagini, K.M. Lampropoulos, S.A. Papadodima, and D.C. Iliopoulos. Biomechanical response of ascending thoracic aortic aneurysms: association with structural remodelling. Comput Methods Biomech Biomed Engin, 15(3):231-48, 2012.

47. O. Trabelsi, F.M. Davis, J.F. Rodriguez-Matas, A. Duprey, and S. Avril. Patient specific stress and rupture analysis of ascending thoracic aneurysms. J Biomech, 48(10):1836-43, 2015.

48. O. Trabelsi, M. Gutierrez, S. Farzaneh, A. Duprey, and S. Avril. A non-invasive technique for ATAA rupture risk estimation. Journal of Biomechanics, page doi.org/10.1016/j.jbiomech.2017.11.012, 2017.

49. EM. van Disseldorp, N.J. Petterson, MC. Rutten, FN. van de Vosse, MR. van Sambeek, and RG. Lopata. Patient specific wall stress analysis and mechanical characterization of abdominal aortic aneurysms using 4D ultrasound. Eur J Vasc Endovasc Surg, 52:635-42, 2016. 
50. D.A. Vorp, B.J. Schiro, M.P. Ehrlich, T.S. Juvonen, M.A. Ergin, and B.P. Griffith. Effect of aneurysm on the tensile strength and biomechanical behavior of the ascending thoracic aorta. Ann Thorac Surg, 75:1210-14, 2003.

51. A. Wittek, K. Karatolios, P. Bihari, T. Schmitz-Rixen, R. Moosdorf, S. Vogt, and C. Blase. In vivo determination of elastic properties of the human aorta based on $4 \mathrm{D}$ ultrasound data. J Mech Behav Biomed Mater, 27:167-183, 2013.

52. A. Wittek, K. Karatolios, C.P. Fritzen, J. Bereiter-Hahn, B. Schieffer, R. Moosdorf, S. Vogt, and C. Blase. Cyclic three-dimensional wall motion of the human ascending and abdominal aorta characterized by time-resolved three-dimensional ultrasound speckle tracking. Biomech Model Mechanobiol, 15(5):1375-88, 2016. 


\section{List of Tables}

1 Demographic information of three patients. Note that $\Delta P$ is the difference between diastolic and systolic pressures, $E h_{\text {in-vivo }}$ denotes the extensional stiffness obtained by [48] and Ehin-vitro is the extensional stiffness obtained by [13]. . . . . . . . . . .

2 Values of non-linear anisotropic material parameters for homoge-

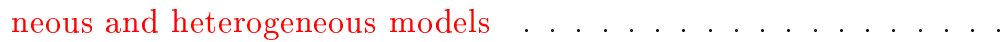


${ }_{725}$ List of Tables 
Table 1 Demographic information of three patients. Note that $\Delta P$ is the difference between diastolic and systolic pressures, Ehin-vivo denotes the extensional stiffness obtained by [48] and $E h_{\text {in-vitro }}$ is the extensional stiffness obtained by [13].

\begin{tabular}{llllll}
\hline $\begin{array}{l}\text { Patient } \\
\text { ID }\end{array}$ & Sex & Age & $\begin{array}{l}\Delta P \\
{[\mathrm{KPa}](\mathrm{mmHg})}\end{array}$ & $\begin{array}{l}\text { Eh } h_{\text {in-vivo }} \\
{[\mathrm{MPa} . \mathrm{mm}]}\end{array}$ & $\begin{array}{l}\text { Eh } h_{\text {in-vitro }} \\
{[\mathrm{MPa} . \mathrm{mm}]}\end{array}$ \\
\hline \hline 1 & $\mathrm{M}$ & 27 & $8.66(65)$ & 1.96 & 0.78 \\
2 & $\mathrm{M}$ & 84 & $13.4(100.5)$ & 6.14 & 4.11 \\
3 & $\mathrm{M}$ & 61 & $11.5(86)$ & 4.6 & 1.24 \\
\hline
\end{tabular}

Table 2 Values of non-linear anisotropic material parameters for homogeneous and heterogeneous models

\begin{tabular}{lccccc}
\hline & $C_{10}[\mathrm{kPa} \cdot \mathrm{mm}]$ & $k_{1}[\mathrm{kPa} . \mathrm{mm}]$ & $k_{2}$ & $\theta_{1}\left[^{\circ}\right]$ & $\theta_{2}\left[{ }^{\circ}\right]$ \\
\hline \hline Homogenous & 30 & 500 & 5 & 35 & -35 \\
Heterogeneous stiff & 60 & 1000 & 5 & 35 & -35 \\
Heterogeneous compli- & 30 & 500 & 5 & 35 & -35 \\
ant & & & & & \\
\hline
\end{tabular}




\section{List of Figures}

1 Segmentation of the aortic geometry for diastolic phase. Including left subclavian artery, brachiocephalic artery, left common carotid artery and coronary artery branches which are used as anatomical references. See video 1 for ten phases of cardiac cycle. . . . . . .

2 It is assumed that each element of the arterial wall is a finite sector of an ellipsoidal membrane with radii $r_{1}$ and $r_{2}$ in both circumferential and longitudinal directions. $n_{1}$ and $n_{2}$ are tangential unit vectors normal to $r_{1}$ and $r_{2}$, respectively. $n_{i}^{n}$ with $\mathrm{i}=\{1,2, \ldots, 4\}$ is normal unit vector at each node of the element. . . . . . . . . . 33

3 Flowchart of the methodology to identify local membrane stiffness

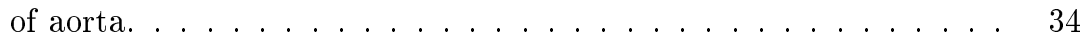

4 Map of the displacement magnitude [mm] after inflation of the reference geometry (RG) using $\mathrm{FE}$ analysis considering a- a homogenous stiffness and b- a heterogeneous stiffness distributions. . . . . . . 35

5 a- Displacement maps [mm] obtained by our methodology considering a homogenous stiffness, b- Reference displacement maps obtained using a finite element analysis considering a homogenous stiffness, c- Displacement maps obtained by our methodology considering a heterogeneous stiffness distribution, d- Reference displacement maps obtained using a finite element analysis considering a heterogeneous stiffness distribution. . . . . . . . . . 36

6 Validation study: stiffness map [MPa.mm] reconstructed using our methodology (left) for the assigned homogeneous stiffness (right)(a). Stiffness map reconstructed using our methodology (left) for the assigned heterogeneous stiffness (right)(b) . . . . . . . . . . . 37

7 Validation study: stiffness map [MPa.mm] reconstructed using our methodology considering a- homogeneous and b- heterogeneous nonlinear anisotropic material properties. . . . . . . . . . .

8 Distribution of local membrane stiffness [MPa.mm] in ascending and descending aortas for Patient 1 (a), Patient 2 (b) and Patient 3 (c). Left and right show frontal and distal views respectively. . . . . . 39 
9 Distributions of local membrane stiffness [MPa.mm] for Patient 1 (a), Patient 2 (b) and Patient 3 (c). Results in the left shows frontal and distal views of in vivo. Results in the right demonstrates in vitro

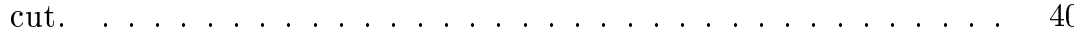

10 Median and interquartile range of the local membrane stiffness [MPa.mm] obtained for in vivo cut, in vitro cut and whole ascending for Patient 1 (a), Patient 2 (b) and Patient 3 (c). The blue and the red stars demonstrate the values of in vivo and in vitro cuts, respectively, which are calculated by [48] and [13] . . . . . . . . . . 41 


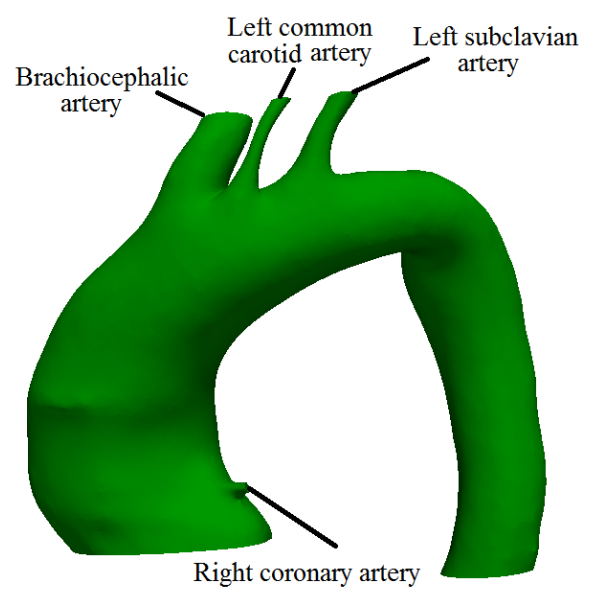

Fig. 1 Segmentation of the aortic geometry for diastolic phase. Including left subclavian artery, brachiocephalic artery, left common carotid artery and coronary artery branches which are used as anatomical references. See video 1 for ten phases of cardiac cycle. 


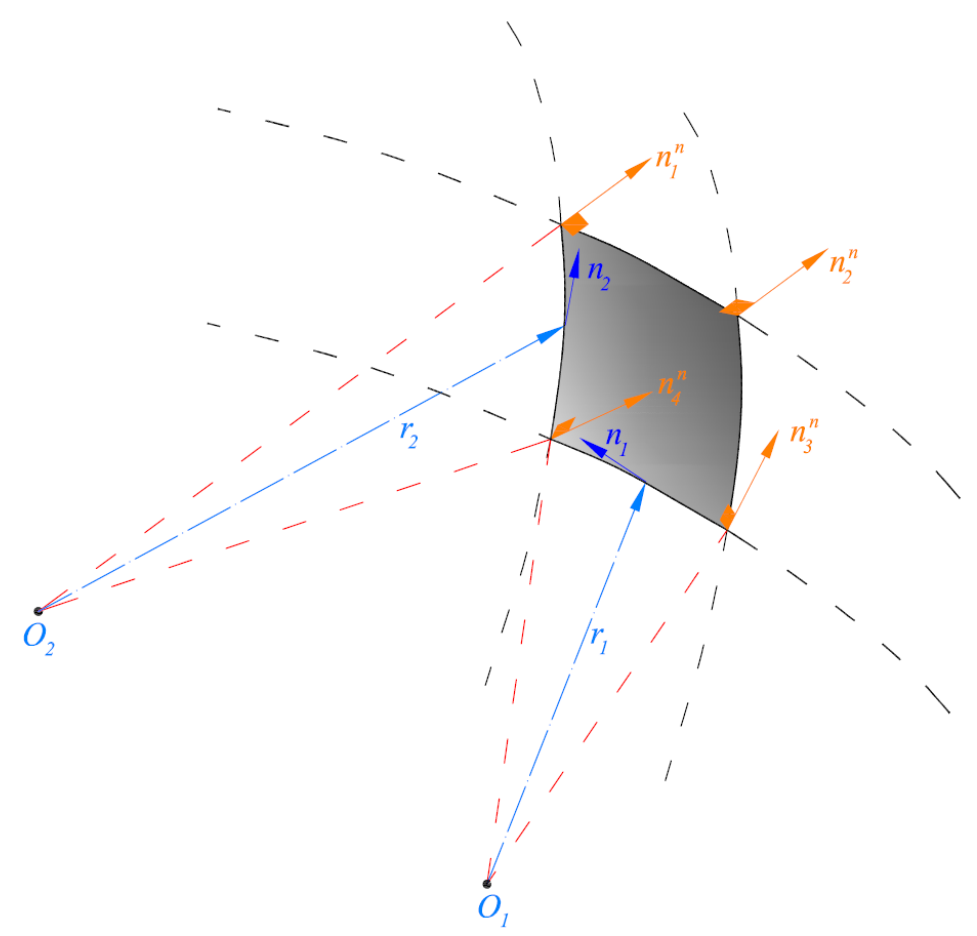

Fig. 2 It is assumed that each element of the arterial wall is a finite sector of an ellipsoidal membrane with radii $r_{1}$ and $r_{2}$ in both circumferential and longitudinal directions. $n_{1}$ and $n_{2}$ are tangential unit vectors normal to $r_{1}$ and $r_{2}$, respectively. $n_{i}^{n}$ with $\mathrm{i}=\{1,2, \ldots, 4\}$ is normal unit vector at each node of the element. 


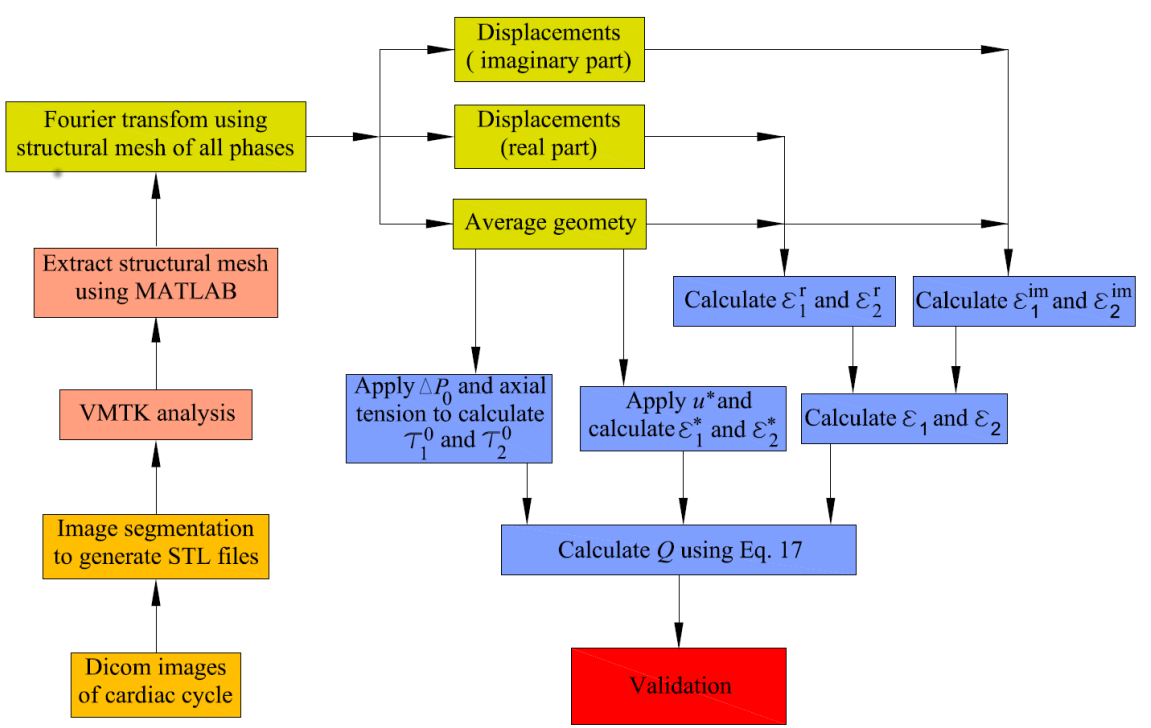

Fig. 3 Flowchart of the methodology to identify local membrane stiffness of aorta. 


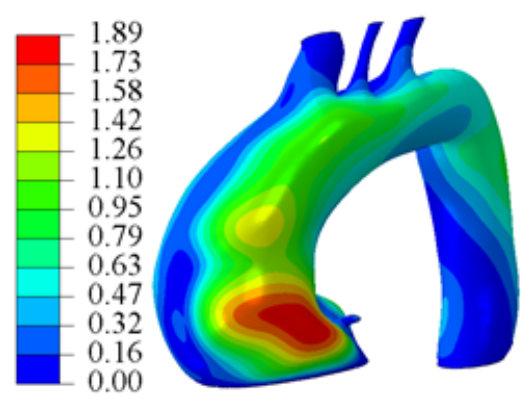

(a)

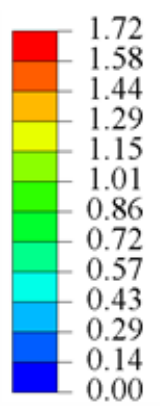

(b)

Fig. 4 Map of the displacement magnitude [mm] after inflation of the reference geometry (RG) using FE analysis considering a- a homogenous stiffness and b- a heterogeneous stiffness distributions. 

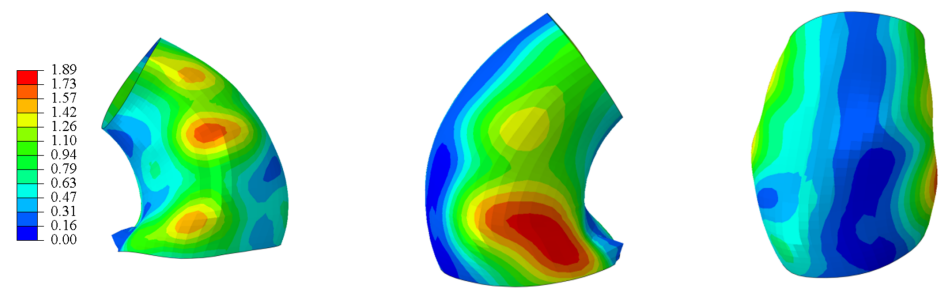

(a)
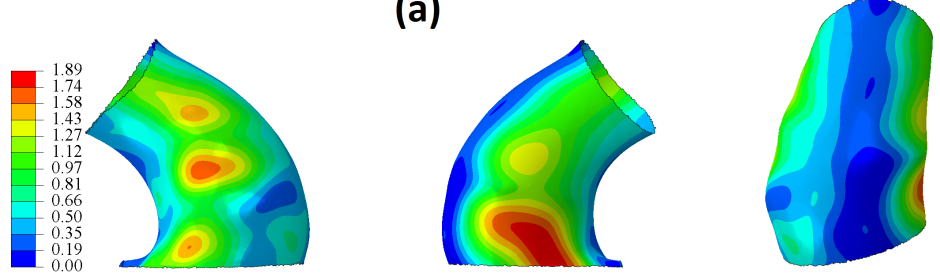

(b)
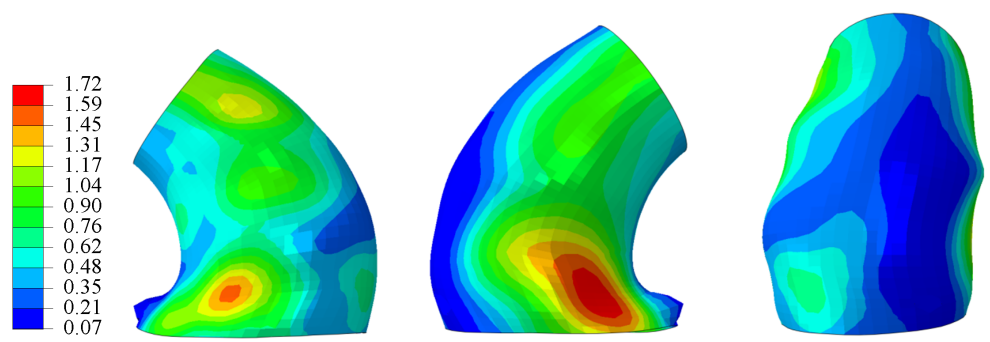

(c)
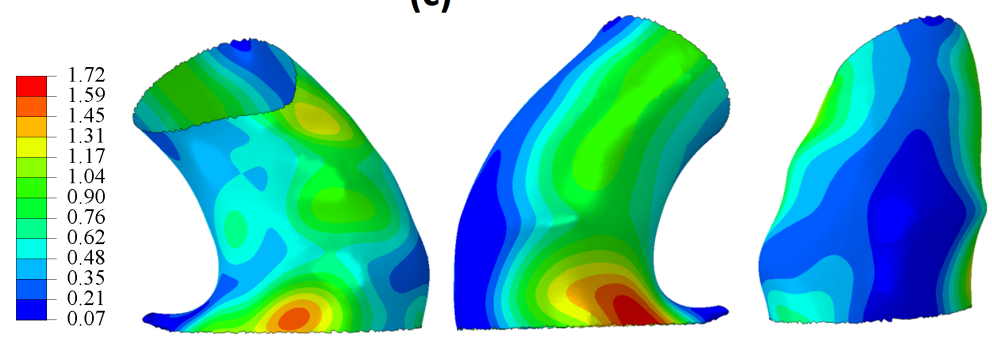

(d)

Fig. 5 a- Displacement maps [mm] obtained by our methodology considering a homogenous stiffness, b- Reference displacement maps obtained using a finite element analysis considering a homogenous stiffness, c- Displacement maps obtained by our methodology considering a heterogeneous stiffness distribution, d- Reference displacement maps obtained using a finite element analysis considering a heterogeneous stiffness distribution. 

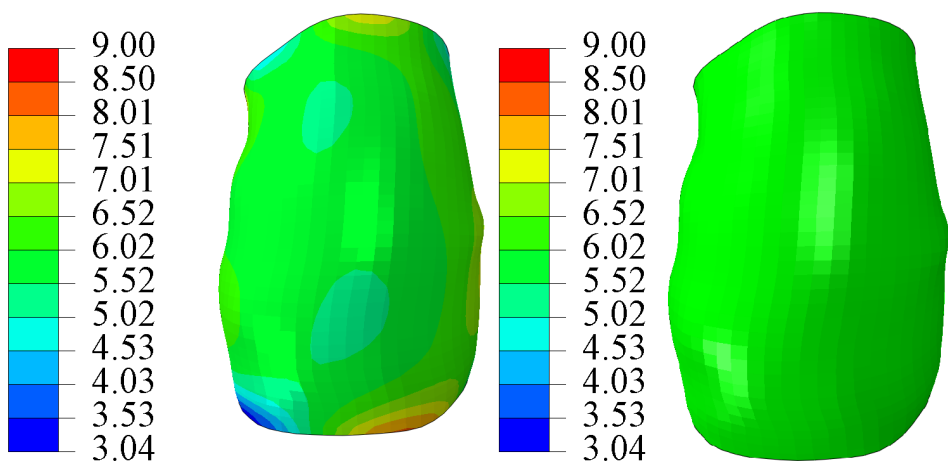

(a)
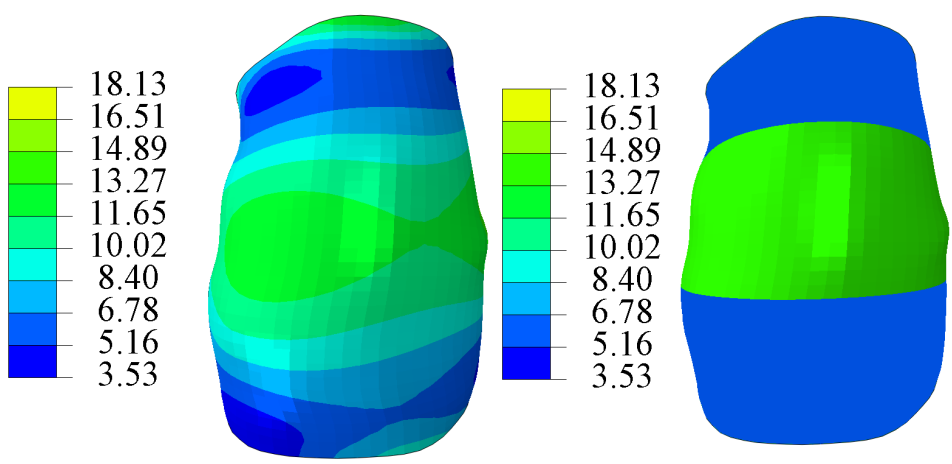

(b)

Fig. 6 Validation study: stiffness map [MPa.mm] reconstructed using our methodology (left) for the assigned homogeneous stiffness (right)(a). Stiffness map reconstructed using our methodology (left) for the assigned heterogeneous stiffness (right)(b). 


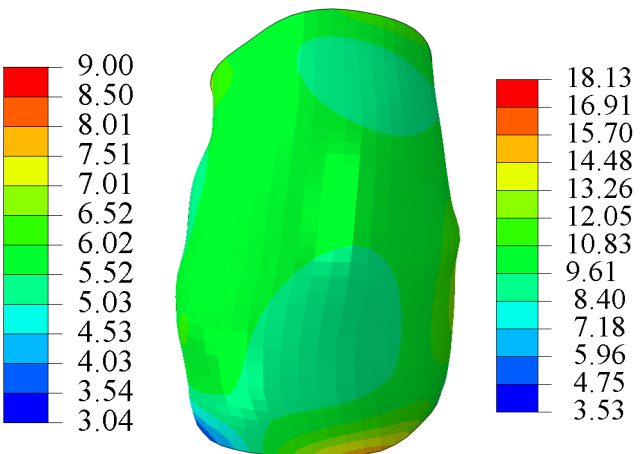

(a)

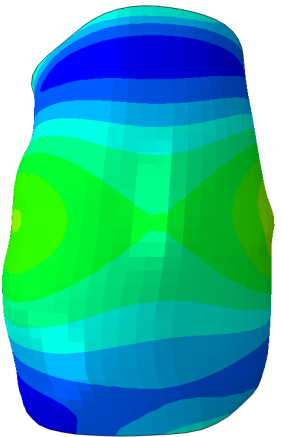

(b)

Fig. 7 Validation study: stiffness map [MPa.mm] reconstructed using our methodology considering a- homogeneous and b- heterogeneous non-linear anisotropic material properties. 

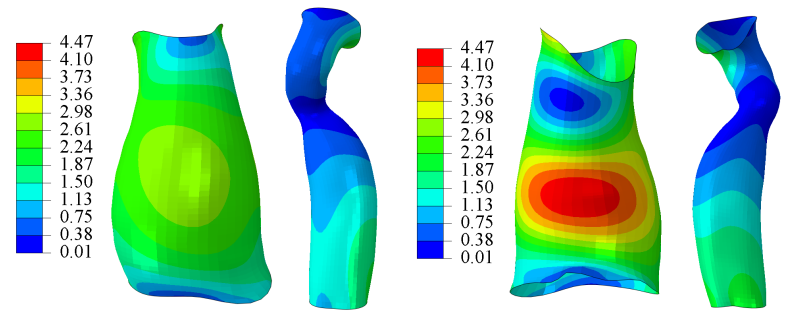

(a)
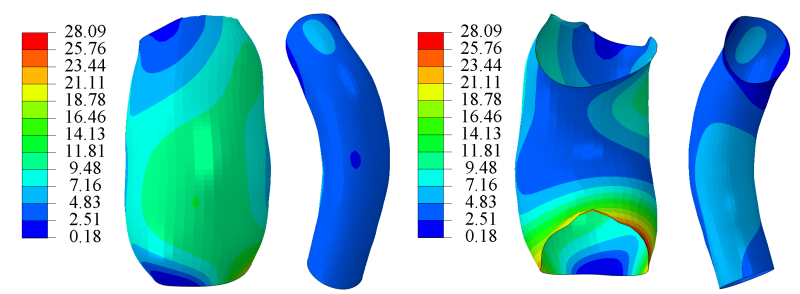

(b)
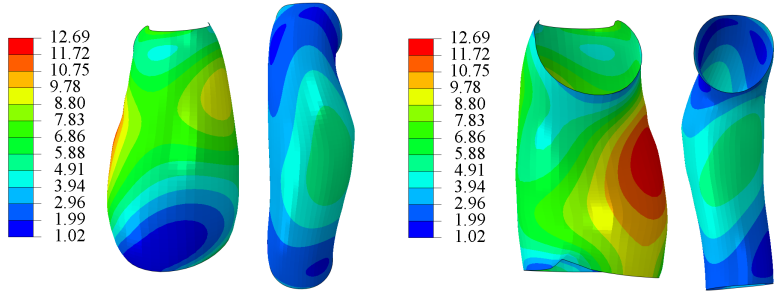

(c)

Fig. 8 Distribution of local membrane stiffness [MPa.mm] in ascending and descending aortas for Patient 1 (a), Patient 2 (b) and Patient 3 (c). Left and right show frontal and distal views respectively. 

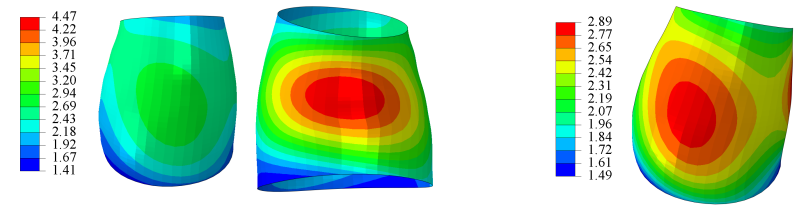

(a)
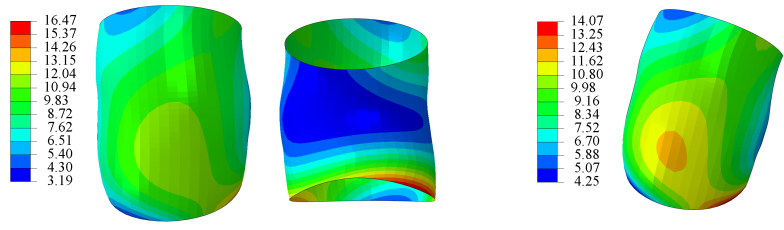

(b)
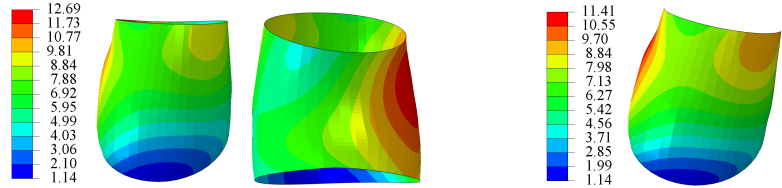

(c)

Fig. 9 Distributions of local membrane stiffness [MPa.mm] for Patient 1 (a), Patient 2 (b) and Patient 3 (c). Results in the left shows frontal and distal views of in vivo. Results in the right demonstrates in vitro cut. 


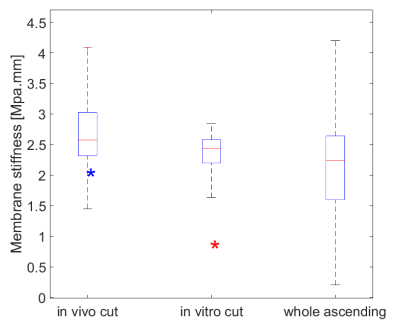

(a)

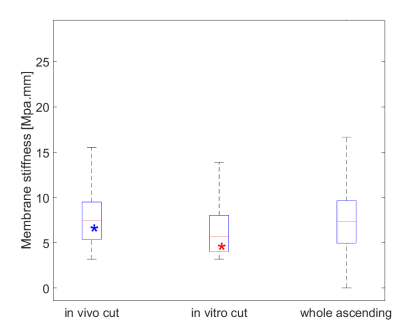

(b)

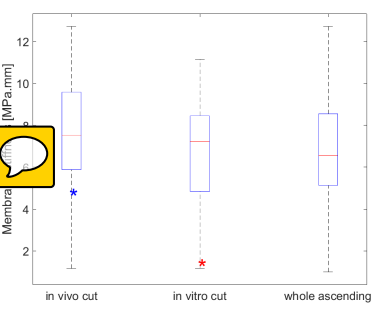

(c)

Fig. 10 Median and interquartile range of the local membrane stiffness [MPa.mm] obtained for in vivo cut, in vitro cut and whole ascending for Patient 1 (a), Patient 2 (b) and Patient 3 (c). The blue and the red stars demonstrate the values of in vivo and in vitro cuts, respectively, which are calculated by [48] and [13]. 